November 2014

\title{
Energy transmutation in nonequilibrium quantum systems
}

\author{
Mihail Mintchev \\ Istituto Nazionale di Fisica Nucleare and Dipartimento di Fisica, Università di Pisa, Largo \\ Pontecorvo 3, 56127 Pisa, Italy \\ Luca Santoni \\ Scuola Normale Superiore, Piazza dei Cavalieri 7, 56126 Pisa, Italy \\ Paul Sorba \\ LAPTh, Laboratoire d'Annecy-le-Vieux de Physique Théorique, CNRS, Université de \\ Savoie, BP 110, 74941 Annecy-le-Vieux Cedex, France
}

\begin{abstract}
We investigate the particle and heat transport in quantum junctions with the geometry of star graphs. The system is in a nonequilibrium steady state, characterized by the different temperatures and chemical potentials of the heat reservoirs connected to the edges of the graph. We explore the Landauer-Büttiker state and its orbit under parity and time reversal transformations. Both particle number and total energy are conserved in these states. However, the heat and chemical potential energy are in general not separately conserved, which gives origin to a basic process of energy transmutation among them. We study both directions of this process in detail, introducing appropriate efficiency coefficients. For scale invariant interactions in the junction our results are exact and explicit. They cover the whole parameter space and take into account all nonlinear effects. The energy transmutation depends on the particle statistics.
\end{abstract}

LAPTH-107/14

IFUP-TH 9/2014 


\section{Introduction}

The study of non-equilibrium quantum systems is among the most rapidly expanding areas of theoretical physics. Triggered by the remarkable experimental progress in manipulating trapped ultra-cold atomic gases, there is recently great interest in the search for universal properties of such systems (see e.g. [1] for a review). Much attention is devoted to the behavior of quantum systems after a quench and in particular, on the nature of the equilibrium state which is approached in this case. Other interesting studies concern the impact of both internal and space-time symmetries (e.g. scale invariance) on the quantum transport in Non-Equilibrium Steady States (NESS).

In this paper we investigate some general features of the energy transport in NESS's, representing non-equilibrium extensions of a Gibbs State (GS). The physical models we focus on are schematically shown in Fig. 1. We are dealing with a multicomponent system represented by the $n \geq 2$ semi-infinite leads (edges) $L_{i}$ of a star graph $\Gamma$. Each lead $L_{i}$ is attached at infinity to a heat reservoir $R_{i}$. The interaction between the $n$ components of the system is localized in the vertex of the graph and is defined by a scattering matrix $\mathbb{S}$. Each heat reservoir $R_{i}$ is described by a GS characterized by inverse temperature $\beta_{i}$ and chemical potential $\mu_{i}$. From Fig. 1 it is evident that the system is away from equilibrium if $\mathbb{S}$ admits a nontrivial transmission coefficient between two reservoirs $R_{i}$ and $R_{j}$ with $\left(\beta_{i}, \mu_{i}\right) \neq\left(\beta_{j}, \mu_{j}\right)$.

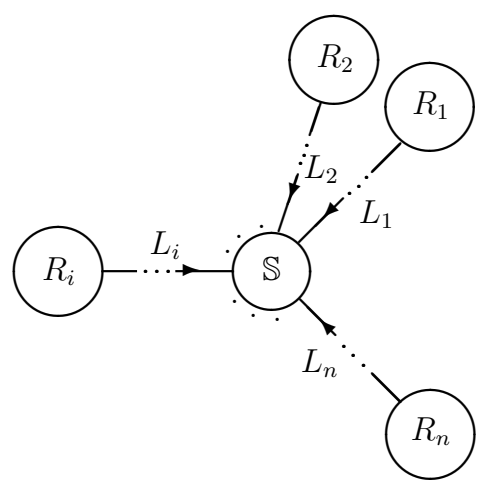

Figure 1: A star graph $\Gamma$ with scattering matrix $\mathbb{S}$ at the vertex and leads $L_{i}$ connected at infinity to thermal reservoirs $R_{i}$.

We start our investigation by shortly reviewing the explicit construction of a NESS $\Omega_{\beta, \mu}$, induced by the scattering matrix $\mathbb{S}$ from the tensor product of the GS's relative to the heat reservoirs $R_{i}$. The state $\Omega_{\beta, \mu}$ is fully determined by $\mathbb{S}$ and $\left(\beta_{i}, \mu_{i}\right)$. We show that $\Omega_{\beta, \mu}$ is not invariant under parity and time-reversal transformations and generates therefore a nontrivial orbit $O_{\beta, \mu}$, consisting of four nonequivalent NESS's. The total energy of the system in these states has two components: heat energy (parametrized by $\beta_{i}$ ) and chemical potential energy (parametrized by $\mu_{i}$ ). Provided that the dynamics of the system is invariant under time translations, the total energy is conserved. For generic values of $\left(\beta_{i}, \mu_{i}\right)$ the two energy components are however not separately conserved and an energy transmutation occurs. The process is controlled by a single parameter $\dot{Q}$, describing the heat flow in the junction. For 
$\dot{Q}<0$ heat energy is transformed in chemical potential energy. The opposite transformation takes place for $\dot{Q}>0$.

The regime $Q<0$ has been investigated mostly by means of linear response theory. For the details we refer to the review paper [2] and the references therein, observing only that in these studies the junction is usually compared to a heat engine, whose efficiency represents the main point of interest. This comparison is very suggestive, keeping always in mind that the junction acts actually as a converter between heat and chemical potential energy. The case $\dot{Q}>0$ is quite subtle and has been explored only partially in the domain where the junction can be compared to a refrigerator or heat pump. The main goal of the present paper is to study the system in Fig. 1 as an energy converter, thus providing a systematic and unified description of both regimes $\dot{Q} \lessgtr 0$, which are characterized by appropriate efficiency coefficients. Since the energy transport in the junction is strongly influenced by nonlinear phenomena, the well known linear response approximation is not suitable for our investigation because it covers only a little part of the parameter space. For this reason a fundamental objective of our study is to simplify as much as possible the dynamics in order to solve the problem in exact form, preserving at the same time a nontrivial quantum transport. In this respect we demonstrate that assuming free propagation along $L_{i}$ and scale-invariant point-like interactions in the vertex is enough for this purpose. We study both fermionic and bosonic systems, showing that the particle statistics affects the energy transmutation. The detailed comparison between the two cases is very instructive, because the bosonic case is poorly investigated. The impact of parity and time reversal on the quantum transport is discussed in detail as well.

We proceed as follows. In the next section we briefly recall the construction of the NESS $\Omega_{\beta, \mu}$ and its orbit $O_{\beta, \mu}$ under parity and time reversal transformations. In section 3 we fix the Schrödinger dynamics on the leads and the interaction at the vertex of $\Gamma$. Studying the local conserved currents and the relative densities, we show that the junction converts heat energy in chemical potential energy or vice versa, depending on the values of the parameters characterizing the reservoirs $R_{i}$. The basic features of this process of energy transmutation are investigated in section 4 , where we introduce the efficiency coefficients for both cases $\dot{Q} \lessgtr 0$. We describe here also the scale invariant (critical) interactions in the junction. The efficiency of the quantum transport for a system with two leads is investigated in section 5 both for the LB state $\Omega_{\beta, \mu}$ and the orbit $O_{\beta, \mu}$. We also relate here our approach to some exact results, concerning other systems and NESS's. In section 6 we describe the main features of a junction with 3 leads. Section 7 is devoted to the bosonic case. The conclusions and some future developments form the content of Section 8. The appendices collect some technical results about the Onsager matrix and the 3-lead junction.

\section{Non equilibrium states on $\Gamma$}

Following the pioneering work of Landauer [3] and Büttiker [4], non-equilibrium systems of the type in Fig. 1 have been extensively investigated. In this section we briefly recall an explicit quantum field theory construction [5] of the Landauer-Büttiker (LB) state $\Omega_{\beta, \mu}$, which 
adapts to the star graph case some general ideas [6]-[10] about NESS's. This construction is very convenient for deriving correlation functions and for studying the orbit $O_{\beta, \mu}$ of $\Omega_{\beta, \mu}$ under parity and time reversal transformations. As mentioned in the introduction, the elements of this orbit represent new nonequivalent NESS's with interesting physical properties.

The first step in constructing the LB state $\Omega_{\beta, \mu}$ is to describe the asymptotic dynamics at $t=-\infty$ (i.e. before the interaction) in terms of the operators

$$
\left\{a_{i}^{*}(k), a_{i}(k): k>0, i=1, \ldots, n\right\},
$$

which create and annihilate the particle excitations with momentum $k$ in the reservoir $R_{i}$. In agreement with the orientation of the leads in Fig.1, the condition $k>0$ implies that (2.1) create and annihilate incoming particles. For fermionic systems (2.1) generate therefore an incoming Canonical Anti-commutation Relations (CAR) algebra $\mathcal{A}^{\text {in }}$. We denote by $\Omega_{\beta_{i}, \mu_{i}}$ be the GS associated with the heat reservoir $R_{i}$ (see e.g. [11]) and consider the tensor product $\otimes_{i=1}^{n} \Omega_{\beta_{i}, \mu_{i}}$.

The next step is to relate $\mathcal{A}^{\text {in }}$ with the CAR algebra of outgoing excitations $\mathcal{A}^{\text {out }}$, generated still by the creation and annihilation operators (2.1), but with $k<0$. For this purpose we have to introduce the interaction, connecting different heat reservoirs and driving the system away from equilibrium. We consider the simple case where the incoming particles propagate freely along the leads towards the vertex of the graph, where they are reflected or transmitted with some probability in the rest of the graph. This process is codified in the reflection-transmission equations

$$
a_{i}(k)=\sum_{j=1}^{n} \mathbb{S}_{i j}(k) a_{j}(-k), \quad a_{i}^{*}(k)=\sum_{j=1}^{n} a_{j}^{*}(-k) \mathbb{S}_{j i}^{*}(k) \quad k<0,
$$

which relate $\mathcal{A}^{\text {in }}$ and $\mathcal{A}^{\text {out }}$. Here $\mathbb{S}(k)$ is the scattering matrix describing the point-like interaction in the vertex of the graph. We assume unitarity and Hermitian analyticity

$$
\mathbb{S}(k) \mathbb{S}(k)^{*}=\mathbb{I}, \quad \mathbb{S}(k)^{*}=\mathbb{S}(-k),
$$

the star * indicating Hermitian conjugation. These conditions imply that $\mathbb{S}(k) \mathbb{S}(-k)=\mathbb{I}$, which ensures the consistency of the constraints (2.2) .

The whole algebra $\mathcal{A}$, generated by polynomials involving generators of both $\mathcal{A}^{\text {in }}$ and $\mathcal{A}^{\text {out }}$, is a deformed 11 CAR algebra, where $\left[a_{i}(k), a_{j}(p)\right]_{+}=\left[a_{i}^{*}(k), a_{j}^{*}(p)\right]_{+}=0$ and

$$
\left[a_{i}(k), a_{j}^{*}(p)\right]_{+}=2 \pi\left[\delta(k-p) \delta_{i j}+\mathbb{S}_{i j}(k) \delta(k+p)\right] .
$$

Because of (2.3), the right hand side of (2.4) defines the kernel of an integral projection (instead of the usual identity) operator. Hermitian analyticity implies [12] that the $*$-operation in $\mathcal{A}$ is a conjugation.

At this point the LB state $\Omega_{\beta, \mu}$ is the extension of $\otimes_{i=1}^{n} \Omega_{\beta_{i}, \mu_{i}}$ from $\mathcal{A}^{\text {in }}$ to the whole algebra $\mathcal{A}$, performed by linearity via the reflection-transmission relations (2.2). This construction,

\footnotetext{
${ }^{1} \mathrm{CAR}$ algebra deformations of the type (2.4) have been studied previously in the context of onedimensional integrable systems with boundaries [12] or defects [13].
} 
which may look at the first sight a bit abstract, is in fact very efficient for deriving the correlation functions defining the LB representation $\mathcal{H}_{\mathrm{LB}}$ of $\mathcal{A}$. We stress that $\mathcal{H}_{\mathrm{LB}}$ describes non-equilibrium physics and is not equivalent to the Fock and Gibbs representations $\mathcal{H}_{\mathrm{F}}$ and $\mathcal{H}_{\mathrm{G}}$ of $\mathcal{A}$, known [12, 14] from the equilibrium case. Denoting by $(\cdot, \cdot)$ the scalar product in the Hilbert space $\mathcal{H}_{\mathrm{LB}}$, one has [5]

$$
\begin{gathered}
\left(\Omega_{\beta, \mu}, a_{j}^{*}(p) a_{i}(k) \Omega_{\beta, \mu}\right) \equiv\left\langle a_{j}^{*}(p) a_{i}(k)\right\rangle_{\beta, \mu}= \\
2 \pi \delta(k-p)\left[\theta(k) \delta_{i j} d_{i}(k)+\theta(-k) \sum_{l=1}^{n} \mathbb{S}_{i l}(k) d_{l}(-k) \mathbb{S}_{l j}^{*}(k)\right] \\
+2 \pi \delta(k+p)\left[\theta(k) d_{i}(k) \mathbb{S}_{i j}^{*}(-k)+\theta(-k) \mathbb{S}_{i j}(k) d_{j}(-k)\right],
\end{gathered}
$$

were

$$
d_{i}(k)=\frac{\mathrm{e}^{-\beta_{i}\left[\omega_{i}(k)-\mu_{i}\right]}}{1+\mathrm{e}^{-\beta_{i}\left[\omega_{i}(k)-\mu_{i}\right]}}
$$

is the Fermi distribution in the heat reservoir $R_{i}$ with dispersion relation $\omega_{i}(k)$. Notice that the construction allows for different dispersion relations in the different reservoirs.

The explicit form of $\left\langle a_{i}(k) a_{j}^{*}(p)\right\rangle_{\beta, \mu}$ is obtained from (2.5) by the substitution

$$
d_{i}(k) \longmapsto c_{i}(k)=\frac{1}{1+\mathrm{e}^{-\beta_{i}\left[\omega_{i}(k)-\mu_{i}\right]}} .
$$

As well known, employing the CAR algebra, one can express a generic $n$-point correlation function as a polynomial of the two-point correlator (2.5). The non-equilibrium features of the correlation functions are encoded in the mixing of the Fermi distributions, associated with the different heat reservoirs $R_{i}$, via the scattering matrix $\mathbb{S}$, which is evident already from (2.5).

An advantage of the above framework is that it allows to investigate directly the behavior of the LB state $\Omega_{\beta, \mu}$ under parity and time reversal. We first recall that these operations are implemented by a unitary operator $P$ and an anti-unitary operator $T$, acting in the algebra $\mathcal{A}$ in the standard way:

$$
P a_{i}(k) P^{-1}=\chi_{P} a_{i}(-k), \quad T a_{i}^{*}(k) T^{-1}=\chi_{T} a_{i}(-k), \quad\left|\chi_{P}\right|=\left|\chi_{T}\right|=1 .
$$

Apart from the multiplicative phase factors, the action of $P$ and $T$ seems to be the same, but one should remember that $P$ is a linear operator, whereas $T$ is anti-linear. Using the explicit form (2.5) of the two-point function and (2.8), one can explicitly verify that

$$
\left(X \Omega_{\beta, \mu}, a_{j}^{*}(p) a_{i}(k) X \Omega_{\beta, \mu}\right) \equiv\left\langle a_{j}^{*}(p) a_{i}(k)\right\rangle_{\beta, \mu}^{X} \neq\left\langle a_{j}^{*}(p) a_{i}(k)\right\rangle_{\beta, \mu}, \quad X=P, T, P T,
$$

if $\left(\beta_{i}, \mu_{i}\right) \neq\left(\beta_{j}, \mu_{j}\right)$ for some reservoirs. Therefore, $\Omega_{\beta, \mu}$ is not invariant under parity and time reversal and generates the nontrivial orbit

$$
O_{\beta, \mu}=\left\{\Omega_{\beta, \mu}, \Omega_{\beta, \mu}^{X}=X \Omega_{\beta, \mu}: X=P, T, P T\right\}
$$


The property (2.9) captures the fundamental difference of the LB state $\Omega_{\beta, \mu}$ with respect to the Fock vacuum $\Omega_{\mathrm{F}} \in \mathcal{H}_{\mathrm{F}}$ and the Gibbs state $\Omega_{\mathrm{G}} \in \mathcal{H}_{\mathrm{G}}$, which are both $P$ - and $T$ invariant. The result (2.9) suggests also that the four states in the orbit $O_{\beta, \mu}$ have different transport properties, which is confirmed by the analysis in section 5.2 below. Since time reversal symmetry is the quantum counterpart of classical reversibility, one can interpret the breakdown of this symmetry in the LB representation $\mathcal{H}_{\mathrm{LB}}$ as quantum irreversibility.

We conclude by observing that above construction can be easily generalized to bosons by replacing the CAR algebra with a Canonical Commutation Algebra (CCA), which implies the substitution of the Fermi distribution in (2.5) with the Bose distribution:

$$
d_{i}(k) \longmapsto b_{i}(k)=\frac{\mathrm{e}^{-\beta_{i}\left[\omega_{i}(k)-\mu_{i}\right]}}{1-\mathrm{e}^{-\beta_{i}\left[\omega_{i}(k)-\mu_{i}\right]}} .
$$

As one can expect on general grounds and as shown in section 7 , the quantum transport is influenced by the statistics.

\section{The Schrödinger junction}

Quantum systems away from equilibrium behave usually in a complicated way. In most of the cases the linear response or other approximations are not enough for fully describing the complexity of this behavior. For this reason the existence of models which incorporate the main non-equilibrium features, while being sufficiently simple to be analyzed exactly, is conceptually very important. An interesting family of such models in $s$ space dimensions is characterized by requiring that the interaction, which drives the system away from equilibrium, is localized on a $(s-1)$-dimensional sub-manifold, whereas the propagation in the complementary orthogonal direction is free. In what follows we will consider a special case of this scenario, focussing on a one-dimensional space with the geometry of a star graph $\Gamma$. Each point $P \in \Gamma$ is parametrized by the coordinates $\{(x, i),: x<0, i=1, \ldots, N\}$, where $|x|$ is the distance of $P$ from the vertex and $i$ labels the lead. Since $s=1$, the interaction is implemented by a point-like defect in the vertex of $\Gamma$ and the propagation along the leads $L_{i}$ is free. We assume in this paper that it is governed by the Schrödinger equation

$$
\left(\mathrm{i} \partial_{t}+\frac{1}{2 m} \partial_{x}^{2}\right) \psi(t, x, i)=0
$$

but other types of time evolution can be considered [5, 15] in the same way. The field $\psi$ is complex and the system has a global $U(1)$-invariance, generating particle number conservation. In the fermionic case $\psi$ satisfies the standard equal-time CAR's.

The scattering matrix $\mathbb{S}$ in the vertex is fixed by requiring that the bulk Hamiltonian $-\partial_{x}^{2}$ admits a self-adjoint extension on the whole graph. These extensions are defined [16]-[18] by

$$
\lim _{x \rightarrow 0^{-}} \sum_{j=1}^{n}\left[\lambda(\mathbb{I}-\mathbb{U})_{i j}+\mathrm{i}(\mathbb{I}+\mathbb{U})_{i j} \partial_{x}\right] \psi(t, x, j)=0
$$


where $\mathbb{U}$ is a $n \times n$ unitary matrix and $\lambda \in \mathbb{R}$ is a parameter with dimension of mass. Eq. (3.13) guaranties unitary time evolution of the system on the graph. The matrices $\mathbb{U}=\mathbb{I}$ and $\mathbb{U}=-\mathbb{I}$ define the Neumann and Dirichlet boundary conditions respectively. The explicit form of the scattering matrix is [16]-[18]

$$
\mathbb{S}(k)=-\frac{[\lambda(\mathbb{I}-\mathbb{U})-k(\mathbb{I}+\mathbb{U})]}{[\lambda(\mathbb{I}-\mathbb{U})+k(\mathbb{I}+\mathbb{U})]} .
$$

The diagonal element $\mathbb{S}_{i i}(k)$ represents the reflection amplitude from the vertex on the lead $L_{i}$, whereas $\mathbb{S}_{i j}(k)$ with $i \neq j$ equals the transmission amplitude from $L_{i}$ to $L_{j}$. One easily verifies that (3.14) satisfies (2.3) and therefore defines an algebra $\mathcal{A}$ of the type introduced in the previous section. Moreover, $\mathbb{S}(k)$ is a meromorphic function in the complex $k$-plane with finite number of simple poles on the imaginary axis. For simplicity we consider in this paper the case without bound states (poles in the upper half plane), referring for the general case to [14, [19]. In this case the solution of equation (3.12) is fixed uniquely by (3.13) and takes the following simple form

$$
\psi(t, x, i)=\sum_{j=1}^{n} \int_{0}^{\infty} \frac{d k}{2 \pi} \mathrm{e}^{-\mathrm{i} \omega(k) t}\left[\mathrm{e}^{-\mathrm{i} k x} \delta_{i j}+\mathrm{e}^{\mathrm{i} k x} \mathbb{S}_{i j}(-k)\right] a_{j}(k), \quad \omega(k)=\frac{k^{2}}{2 m}
$$

As expected, the parity and time-reversal transformations (2.8) in the algebra $\mathcal{A}$ imply

$$
P \psi(t, x, i) P^{-1}=\chi_{P} \psi(t,-x, i), \quad T \psi(t, x, i) T^{-1}=\chi_{T} \psi(-t, x, i) .
$$

Let us describe now the basic local observables, whose behavior away from equilibrium is the main topic of this paper. The local particle density and relative current are

$$
j_{t}(t, x, i)=\left[\psi^{*} \psi\right](t, x, i), \quad j_{x}(t, x, i)=\frac{\mathrm{i}}{2 m}\left[\psi^{*}\left(\partial_{x} \psi\right)-\left(\partial_{x} \psi^{*}\right) \psi\right](t, x, i),
$$

The total energy density is

$$
\theta_{t t}(t, x, i)=-\frac{1}{4 m}\left[\psi^{*}\left(\partial_{x}^{2} \psi\right)+\left(\partial_{x}^{2} \psi^{*}\right) \psi\right](t, x, i),
$$

with energy flow

$$
\theta_{x t}(t, x, i)=\frac{1}{4 m}\left[\left(\partial_{t} \psi^{*}\right)\left(\partial_{x} \psi\right)+\left(\partial_{x} \psi^{*}\right)\left(\partial_{t} \psi\right)-\left(\partial_{t} \partial_{x} \psi^{*}\right) \psi-\psi^{*}\left(\partial_{t} \partial_{x} \psi\right)\right](t, x, i) .
$$

The equations of motion lead to the local conservation laws

$$
\left(\partial_{t} j_{t}-\partial_{x} j_{x}\right)(t, x, i)=\left(\partial_{t} \theta_{t t}-\partial_{x} \theta_{x t}\right)(t, x, i)=0
$$

The relation between local conservation laws and the associated charges on a star graph has been investigated in [20]. In the presence of a defect, like the junction in our case, the 
local conservation (3.20) alone is not enough [20] to ensure the conservation of the relative quantum numbers. One needs in addition the Kirchhoff rules

$$
\sum_{i=1}^{n} j_{x}(t, 0, i)=0, \quad \sum_{i=1}^{n} \theta_{x t}(t, 0, i)=0 .
$$

It is worth stressing that the explicit form (3.14) of the $\mathbb{S}$-matrix is fundamental for proving [5] the operator form (3.21) of the Kirchhoff rules, which guaranties the charge conservation in the whole state space $\mathcal{H}_{\mathrm{LB}}$ of the system. Combining (3.20) and (3.21) one concludes that for these $\mathbb{S}$-matrices the particle number and the total energy in our system are conserved.

The heat density $q_{t}$ in the lead $L_{i}$ is obtained (see e.g. [21]) by subtracting from the total energy density the energy density relative to the chemical potential $\mu_{i}$, namely

$$
q_{t}(t, x, i)=\theta_{t t}(t, x, i)-\mu_{i} j_{t}(t, x, i)
$$

Accordingly, the heat current is

$$
q_{x}(t, x, i)=\theta_{x t}(t, x, i)-\mu_{i} j_{x}(t, x, i)
$$

Local heat conservation

$$
\left(\partial_{t} q_{t}-\partial_{x} q_{x}\right)(t, x, i)=0
$$

is a direct consequence of (3.20), but in general the relative Kirchhoff rule is not at all satisfied. In fact, a key observation is that the heat current obeys the operator Kirchhoff rule if and only if $\mu_{i}=\mu_{j}$ for all $i, j=1, \ldots, N$. Otherwise, the heat current violates the Kirchhoff rule and the heat energy is therefore not conserved. The chemical potential energy shares the same property, because the total energy is conserved.

Summarizing, if $\mu_{i} \neq \mu_{j}$ for some $i$ and $j$, the system converts heat energy in chemical potential energy or vice versa. This is the basic physical process which takes place in the junction. In what follows we will study in detail this phenomenon of energy transmutation.

\section{Transport in the state $\Omega_{\beta, \mu}$}

\subsection{Currents and efficiency}

In order to study the non-equilibrium features of our system, we derive now the expectation values in the state $\Omega_{\beta, \mu}$ of the charge densities and currents introduced above. Since these observables are quadratic, the basic input is the non-equilibrium two-point correlation function

$$
\begin{gathered}
\left\langle\psi^{*}\left(t_{1}, x_{1}, i\right) \psi\left(t_{2}, x_{2}, j\right)\right\rangle_{\beta, \mu}=\int_{0}^{\infty} \frac{\mathrm{d} k}{2 \pi} \mathrm{e}^{\mathrm{i} \omega(k) t_{12}}\left[\delta_{j i} d_{i}(k) \mathrm{e}^{\mathrm{i} k x_{12}}+\right. \\
\left.d_{j}(k) \mathbb{S}_{j i}(k) \mathrm{e}^{-\mathrm{i} k \widetilde{x}_{12}}+\mathbb{S}_{j i}^{*}(k) d_{i}(k) \mathrm{e}^{\mathrm{i} k \widetilde{x}_{12}}+\sum_{l=1}^{n} \mathbb{S}_{j l}^{*}(k) d_{l}(k) \mathbb{S}_{l i}(k) \mathrm{e}^{-\mathrm{i} k x_{12}}\right],
\end{gathered}
$$


following from (2.5/3.15). Here $t_{12}=t_{1}-t_{2}, x_{12}=x_{1}-x_{2}$ and $\widetilde{x}_{12}=x_{1}+x_{2}$. The invariance of (4.25) under time translations explicitly confirms that the total energy of the system is conserved. Combining (4.25) with (3.17,3.19) and performing the limits $t_{1} \rightarrow t_{2}=t$ and $x_{1} \rightarrow x_{2}=x$ one gets the following current expectation values

$$
\begin{gathered}
J_{i}^{N} \equiv\left\langle j_{x}(t, x, i)\right\rangle_{\beta, \mu}=\sum_{j=1}^{n} \int_{0}^{\infty} \frac{\mathrm{d} k}{2 \pi} \frac{k}{m}\left[\delta_{i j}-\left|\mathbb{S}_{i j}(k)\right|^{2}\right] d_{j}(k), \\
J_{i}^{E} \equiv\left\langle\theta_{t x}(t, x, i)\right\rangle_{\beta, \mu}=\sum_{j=1}^{n} \int_{0}^{\infty} \frac{\mathrm{d} k}{2 \pi} \frac{k}{m}\left[\delta_{i j}-\left|\mathbb{S}_{i j}(k)\right|^{2}\right] \omega(k) d_{j}(k) .
\end{gathered}
$$

The right hand sides of (4.26,4.27) are the Landauer-Büttiker expressions [3, 4, 22, for the particle and energy currents in our case specified by the $\mathbb{S}$ matrix (3.14). This is the main reason for referring to $\Omega_{\beta, \mu}$ as the LB state. Notice that for $\beta_{1}=\cdots=\beta_{n}$ and $\mu_{1}=\cdots=\mu_{n}$ the system is in equilibrium and the currents (4.26, 4.27) vanish due to the unitarity of $\mathbb{S}$.

From (3.23) one gets the heat current

$$
J_{i}^{Q}=J_{i}^{E}-\mu_{i} J_{i}^{N}
$$

As already observed, in general the heat and chemical potential currents $J_{i}^{Q}$ and $\mu_{i} J_{i}^{N}$ do not satisfy separately the Kirchhoff rule. In fact, the heat flow $\dot{Q}$ from the junction is given by

$$
\dot{Q}+\sum_{i=1}^{n} J_{i}^{Q}=0 .
$$

Taking into account the Kirchhoff rule (3.21) for the energy current one gets

$$
\dot{Q}=-\sum_{i=1}^{n} J_{i}^{Q}=\sum_{i=1}^{n} \mu_{i} J_{i}^{N},
$$

If $\dot{Q}<0$ the junction transforms heat energy in chemical potential energy. The efficiency of this process can be characterized as follows. Let us denote by $\mathcal{K}_{\text {out }}$ the subset of heat currents leaving the reservoirs $R_{i}$. With our choice for the orientation of the leads (see Fig. 1), these currents are positive. With this convention the efficiency of the junction to transform heat energy in chemical potential energy is defined by

$$
\eta=\frac{\sum_{i=1}^{n} J_{i}^{Q}}{\sum_{i \in \mathcal{K}_{\text {out }}} J_{i}^{Q}}=\frac{-\dot{Q}}{\sum_{i \in \mathcal{K}_{\text {out }}} J_{i}^{Q}},
$$

which is a direct extension of the cases $n=2,3$ [2, 23] to a generic $n$. Combining (4.30) with $\dot{Q}<0$ one concludes that $\sum_{i \in \mathcal{K}_{\text {out }}} J_{i}^{Q}>0$, which implies that $\eta$ is well defined and satisfies $0<\eta \leq 1$.

In the regime $\dot{Q}>0$ of converting chemical potential energy to heat energy the junction is usually compared (see e.g. [2]) to a refrigerator or a heat pump, thus involving in the 
description the known performance coefficients of these devises. We show below that this approach works in parts of the parameter space, but can not be applied globally to the whole domain with $\dot{Q}>0$. In order to avoid this problem, for $\dot{Q}>0$ we propose and adopt below the efficiency

$$
\widetilde{\eta}=\frac{\sum_{i=1}^{n} \mu_{i} J_{i}^{N}}{\sum_{i \in \mathcal{L}_{\text {out }}} \mu_{i} J_{i}^{N}}=\frac{\dot{Q}}{\sum_{i \in \mathcal{L}_{\text {out }}} \mu_{i} J_{i}^{N}},
$$

where now the sum in the denumerator runs over the subset $\mathcal{L}_{\text {out }}$ of outgoing (positive) chemical potential energy currents $\mu_{i} J_{i}^{N}$. The general form of (4.32) is analogous to that of (4.31), but refers to the chemical potential energy. By construction $0<\widetilde{\eta} \leq 1$ in this case as well.

For $\dot{Q}=0$ there is no energy transmutation.

We conclude this subsection by observing that the heat flow in the system generates the following entropy production [21]

$$
\dot{S}=-\sum_{i=1}^{n} \beta_{i} J_{i}^{Q}
$$

Employing (4.26 4.27), $\dot{S}$ can be written in the form

$$
\dot{S}=\sum_{i, j=1}^{n} \int_{0}^{\infty} \frac{\mathrm{d} k}{2 \pi} \frac{k}{m}\left|\mathbb{S}_{i j}(k)\right|^{2}\left[\sigma_{i}(k)-\sigma_{j}(k)\right] d_{j}(k),
$$

with

$$
\sigma_{i}(k)=\beta_{i}\left[\omega(k)-\mu_{i}\right]
$$

Except in section 5.3, we will always assume in this paper that the bulk theory and the heat reservoirs have the same dispersion relation, namely

$$
\omega_{i}(k)=\omega(k) .
$$

With this assumption

$$
d_{j}(k)=\frac{1}{\mathrm{e}^{\sigma_{j}(k)}+1} .
$$

Now, using that (4.37) is a strictly decreasing function of $\sigma_{j}$, one can prove [24] that the integrand of (4.34) is nonnegative 2 , implying the second law of thermodynamics $\dot{S} \geq 0$ in the LB state for all $\left(\beta_{i}, \mu_{i}\right)$ and scattering matrices (3.14). As explained in section 5.2 below, this argument does not apply to the other states of the orbit $O_{\beta, \mu}$ and the entropy production there behaves indeed differently.

Summarizing, the process of energy transmutation in the junction is controlled by the two parameters $\dot{Q}$ and $\dot{S}$ and is characterized by the efficiency coefficients $\eta$ and $\widetilde{\eta}$. We derive in what follows the explicit form of these physical quantities, assuming that the interaction at the junction is scale invariant.

\footnotetext{
${ }^{2}$ The elegant argument of [24] is based on the inequality $F(x)-F(y) \leq(x-y) f(y)$, where $F$ is any primitive of a strictly decreasing function $f$.
} 


\subsection{The scale invariant junction}

The great advantage of considering the non-equilibrium Schrödinger junction defined in section 3 is that it provides both exact and explicit results with nontrivial transport. In order to keep this property also after the integration in $k$, we select in what follows the scaleinvariant elements among the scattering matrices (3.14). They preserve the basic features of the system, while being simple enough to allow the explicit $k$-integration in (4.26, 4.27). The requirement of scale invariance implies that the interaction is $k$-independent and leads to [25]

$$
\mathbb{S}=\mathcal{U} \mathbb{S}_{d} \mathcal{U}^{*}, \quad \mathcal{U} \in U(n), \quad \mathbb{S}_{d}=\operatorname{diag}( \pm 1, \pm 1, \ldots, \pm 1)
$$

The family (4.38) is the orbit of the diagonal matrix $\mathbb{S}_{d}$ under the adjoint action of $U(n)$. We can always enumerate the leads $L_{i}$ in such a way that the first $i$ eigenvalues of $\mathbb{S}$ are +1 and the remaining $n-i$ are -1 . For $i=n$ and $i=0$ one gets $\mathbb{S}=\mathbb{I}$ and $\mathbb{S}=-\mathbb{I}$, which imply vanishing transport because the leads $L_{i}$ are disconnected. One has a nontrivial transport for $0<i<n$.

The scattering matrices of the type (4.38) are in general not symmetric. One can easily prove however that the relative amplitudes are symmetric, namely

$$
\left|\mathbb{S}_{i j}\right|^{2}=\left|\mathbb{S}_{j i}\right|^{2}
$$

This property of the critical points (4.38) simplifies considerably the study of junctions with $n \geq 3$ leads.

The $k$-integration in (4.26) 4.27) with the constant scattering matrices (4.38) can be performed explicitly. One finds

$$
\begin{aligned}
& J_{i}^{N}=\frac{1}{2 \pi} \sum_{j=1}^{n}\left[\delta_{i j}-\left|\mathbb{S}_{i j}\right|^{2}\right] \frac{1}{\beta_{j}} \ln \left(1+\mathrm{e}^{\beta_{j} \mu_{j}}\right), \\
& J_{i}^{E}=-\frac{1}{2 \pi} \sum_{j=1}^{n}\left[\delta_{i j}-\left|\mathbb{S}_{i j}\right|^{2}\right] \frac{1}{\beta_{j}^{2}} \operatorname{Li}_{2}\left(-\mathrm{e}^{\beta_{j} \mu_{j}}\right),
\end{aligned}
$$

where $\mathrm{Li}_{s}$ is the polylogarithm function. These expressions are the building blocks for the parameters $\dot{Q}$ and $\dot{S}$ and the efficiency coefficients $\eta$ and $\widetilde{\eta}$. They depend on $\left(\beta_{i}, \mu_{i}\right)$ and $\left|\mathbb{S}_{i j}\right|^{2}$, which (due to the unitarity of $\mathbb{S}$ ) leads to $n(n+3) / 2$ independent real parameters. We observe in particular that the currents depend on $\mu_{i}$ separately and not on differences $\mu_{i}-\mu_{j}$. In order to reduce this large number it is instructive to start by considering the system with two leads shown in Fig. 2, 


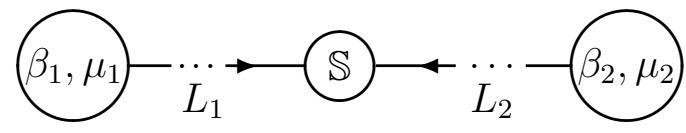

Figure 2: Two heat reservoirs $\left(\beta_{1}, \mu_{1}\right)$ and $\left(\beta_{2}, \mu_{2}\right)$ connected by $\mathbb{S}$.

\section{The case of two leads}

\subsection{The LB state}

In the case $n=2$ there is only one transmission probability $\left|\mathbb{S}_{12}\right|^{2}=\left|\mathbb{S}_{21}\right|^{2}$ and the currents (4.40,4.41) take the form

$$
\begin{aligned}
& J_{1}^{N}=-J_{2}^{N}=\frac{\left|\mathbb{S}_{12}\right|^{2}}{2 \pi \beta_{1}}\left[\ln \left(1+\mathrm{e}^{-\lambda_{1}}\right)-r \ln \left(1+\mathrm{e}^{-\lambda_{2}}\right)\right], \\
& J_{1}^{E}=-J_{2}^{E}=-\frac{\left|\mathbb{S}_{12}\right|^{2}}{2 \pi \beta_{1}^{2}}\left[\operatorname{Li}_{2}\left(-\mathrm{e}^{-\lambda_{1}}\right)-r^{2} \operatorname{Li}_{2}\left(-\mathrm{e}^{-\lambda_{2}}\right)\right],
\end{aligned}
$$

where

$$
r=\frac{\beta_{1}}{\beta_{2}}, \quad \lambda_{i}=-\beta_{i} \mu_{i}, \quad i=1,2 .
$$

The factorization of $\left|\mathbb{S}_{12}\right|^{2}$ implies that the signs of $\dot{Q}$ and $\dot{S}$ depend exclusively on the parameters of the two heat reservoirs $R_{1,2}$, which greatly simplifies the study of the energy transmutation in the junction.

We focus first on $\dot{Q}$ denoting by $\mathcal{D}_{\mp}$ the domains in the space of parameters $\left(\beta_{i}, \mu_{i}\right)$ where $\dot{Q} \lessgtr 0$ respectively. Without loss of generality one can assume $0 \leq r \leq 1$. The direct investigation of

$$
\dot{Q}\left(\lambda_{1}, \lambda_{2}, r\right)=\frac{\left|\mathbb{S}_{12}\right|^{2}}{2 \pi \beta_{1}^{2}}\left(\lambda_{1}-r \lambda_{2}\right)\left[r \ln \left(1+\mathrm{e}^{-\lambda_{2}}\right)-\ln \left(1+\mathrm{e}^{-\lambda_{1}}\right)\right],
$$

shows that

$$
\mathcal{D}_{-}=D_{1} \cup D_{2} \cup D_{3}
$$

with

$$
\begin{aligned}
& D_{1}=\left\{0<\lambda_{1} \leq \lambda_{2}, 0 \leq r<r_{1}\right\} \\
& D_{2}=\left\{\lambda_{1}>\lambda_{2}, \lambda_{1}>0,0 \leq r<r_{2}\right\} \\
& D_{3}=\left\{0 \geq \lambda_{1}>\lambda_{2}, r_{1}<r<r_{2}\right\}
\end{aligned}
$$

where

$$
r_{1}=\frac{\lambda_{1}}{\lambda_{2}}, \quad r_{2}=\frac{\ln \left(1+\mathrm{e}^{-\lambda_{1}}\right)}{\ln \left(1+\mathrm{e}^{-\lambda_{2}}\right)}
$$


The domain $\mathcal{D}_{-}$has a complicated structure due to the dependence of $r_{1,2}$ on $\lambda_{i}$.

We are ready at this point to compute the efficiency $\eta$. Using that $J_{1}^{Q}>0$ and $J_{2}^{Q}<0$ in $\mathcal{D}_{-}$, one gets from (4.31)

$$
\eta\left(\lambda_{1}, \lambda_{2} ; r\right)=\frac{\left(\lambda_{1}-r \lambda_{2}\right)\left[\ln \left(1+\mathrm{e}^{-\lambda_{1}}\right)-r \ln \left(1+\mathrm{e}^{-\lambda_{2}}\right)\right]}{\lambda_{1}\left[\ln \left(1+\mathrm{e}^{-\lambda_{1}}\right)-r \ln \left(1+\mathrm{e}^{-\lambda_{2}}\right)\right]-\left[\operatorname{Li}_{2}\left(-\mathrm{e}^{-\lambda_{1}}\right)-r^{2} \operatorname{Li}_{2}\left(-\mathrm{e}^{-\lambda_{2}}\right)\right]} .
$$

This is an exact and explicit result for the efficiency of the Schrödinger junction in transforming heat to chemical potential energy at criticality. The expression (5.51) shows the power of scale invariance and makes evident the advantage of the above approach with respect to the linear response approximation, which gives information about (5.51) only in the neighborhood of $\lambda_{1} \sim \lambda_{2}$ and $r \sim 1$. We demonstrate in appendix $A$ that in this neighborhood the efficiency (5.51) reproduces exactly the result of the linear response theory in [2].

The analysis of (5.51) in $\mathcal{D}_{-}$shows that the maximal efficiency is obtained in the limit $\lambda_{1}=\lambda_{2} \equiv \lambda \rightarrow+\infty$. In fact one has

$$
\eta_{\max }(r)=\lim _{\lambda \rightarrow+\infty} \eta(\lambda, \lambda ; r)=1-r \equiv \eta_{C},
$$

which is the well known Carnot efficiency. According to (5.45), in this limit the heat energy conversion vanishes $\lim _{\lambda \rightarrow \infty} \dot{Q}(\lambda, \lambda, r)=0$.
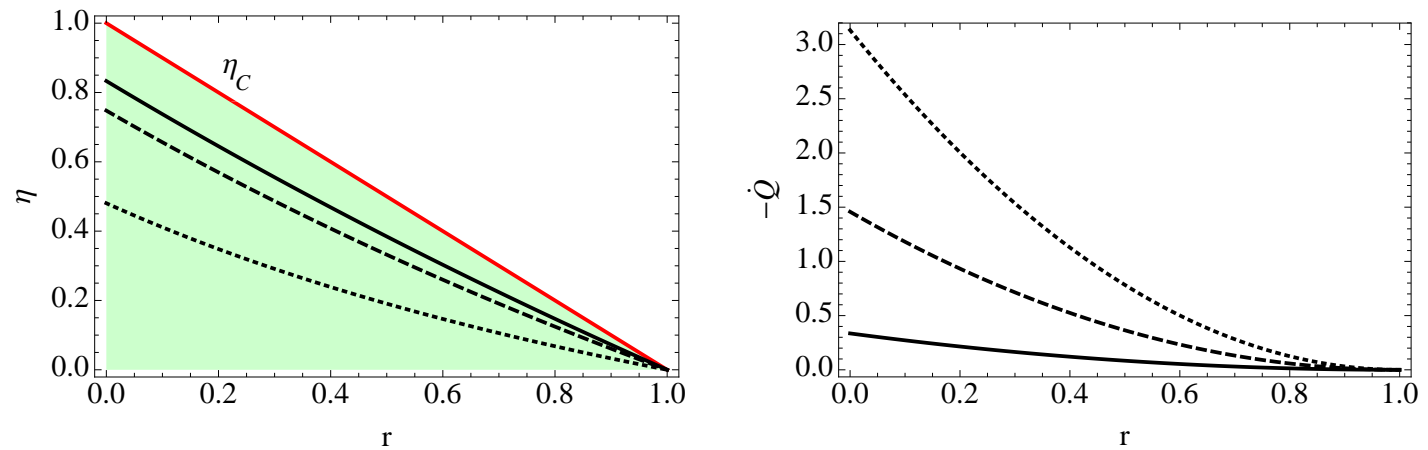

Figure 3: The efficiency $\eta$ compared to $\eta_{C}$ (left) and the heat $-\dot{Q}$ converted to chemical energy (right) with $\lambda=1$ (dotted), $\lambda=3$ (dashed) and $\lambda=5$ (continuous) .

Fig. 3 illustrates the behavior of $\eta$ and the heat converted to chemical energy $-\dot{Q}$ for some values of the parameter $\lambda \equiv \lambda_{1}=\lambda_{2}$. The plots in this figure show that the chemical energy production decreases with increasing the efficiency.

Another physically interesting regime is obtained by maximizing the chemical energy production $-\dot{Q}\left(\lambda_{1}, \lambda_{2}, r\right)$ with respect to $\lambda_{i}$. A simple analysis shows that this function reaches its maximum at $\lambda_{1}=\lambda_{2} \equiv \lambda^{*}$, where

$$
\lambda^{*}-\left(1+\mathrm{e}^{\lambda^{*}}\right) \ln \left(1+\mathrm{e}^{-\lambda^{*}}\right)=0 .
$$

The solution of (5.53) is $\lambda^{*}=1.14455 \ldots$ and the efficiency (5.51) takes the form

$$
\eta^{*}(r) \equiv \eta\left(\lambda^{*}, \lambda^{*} ; r\right)=\frac{(1-r) \lambda^{*} \ln \left(1+\mathrm{e}^{-\lambda^{*}}\right)}{\lambda^{*} \ln \left(1+\mathrm{e}^{-\lambda^{*}}\right)-(1+r) \mathrm{Li}_{2}\left(-\mathrm{e}^{-\lambda^{*}}\right)} .
$$




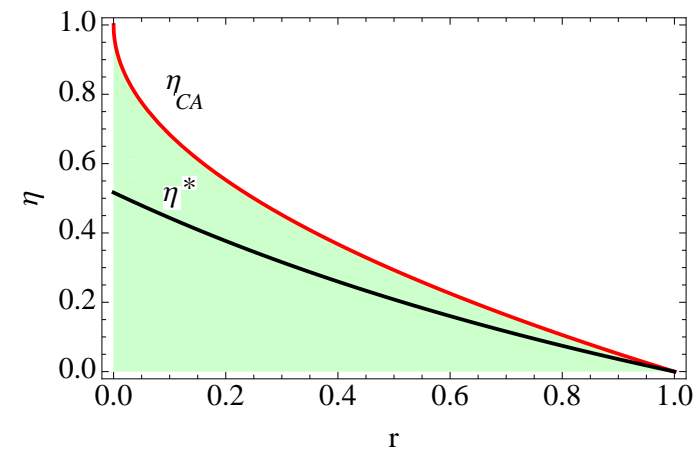

Figure 4: The efficiency $\eta^{*}$ and the Curzon-Ahlborn bound $\eta_{C A}$.

The quantity $\eta^{*}(r)$ is the counterpart of the concept of efficiency at maximal power, used in the context of heat engines. The plot in Fig. 4 shows that (5.54) satisfies the Curzon-Ahlborn bound

$$
\eta^{*}(r) \leq 1-\sqrt{r} \equiv \eta_{C A}
$$

for all $r \in[0,1]$, which was known previously from linear response theory only for $r \sim 1$. We recall that this bound has been proposed for heat engines in the framework of endoreversible thermodynamics in [26]. The rigorous proof [27] covers the linear response regime, but it is known [28, 29, 30] that away of this regime the bound is not universal and can be violated. The possibility to enhance $\eta^{*}$ above $\eta_{C A}$ in our context is discussed in section 5.3.

We turn now to the case when chemical energy is transformed in heat, namely

$$
\dot{Q}=\mu_{1} J_{1}^{N}+\mu_{2} J_{2}^{N}=\left(\mu_{1}-\mu_{2}\right) J_{1}^{N}>0 .
$$

It is convenient to use in the domain $\mathcal{D}_{+}$the coordinates $\left(\beta_{i}, \mu_{i}\right)$. Keeping $\beta_{i}$ arbitrary, one can assume without loss of generality that $\mu_{1}>\mu_{2}$ and set $\mathcal{D}_{+}=\left\{\beta_{1}, \beta_{2}, \mu_{1}>\mu_{2}\right\}$. From (5.56) and the Kirchhoff rule one infers that $J_{1}^{N}>0>J_{2}^{N}$ on $\mathcal{D}_{+}$. Using this information one finds that the efficiency $\widetilde{\eta}$, defined by (4.32), can be expressed in terms of the parameter $u \equiv \mu_{2} / \mu_{1}$ in the simple form

$$
\widetilde{\eta}(u)=\left\{\begin{array}{ll}
1-u, & \mu_{1}>\mu_{2} \geq 0, \\
1, & \mu_{1} \geq 0>\mu_{2}, \\
1-1 / u, & 0>\mu_{1}>\mu_{2},
\end{array} \quad u=\frac{\mu_{2}}{\mu_{1}},\right.
$$

fully covering the domain $\mathcal{D}_{+}$. The formula (5.57) describes in exact form the conversion of chemical potential energy in heat. For $n=2$ the efficiency $\tilde{\eta}$ does not depend on the temperatures and the explicit form of the currents $\mu_{i} J_{i}^{N}$, but only on the values of the chemical potentials $\mu_{i}$. The first line of (5.57) resembles the Carnot formula, where the temperature ratio $r$ is substituted by the chemical potential ratio $u$. The third line instead takes into account that differently from the temperatures, the chemical potentials can take also negative values. Finally, we observe that for $\mu_{1}>0>\mu_{2}$ both chemical potential currents $\mu_{i} J_{i}^{N}$ are flowing towards the junction, transforming the chemical energy in heat completely $(\widetilde{\eta}=1)$. Since in this case the domains of $\mu_{i}$ are separated by the point $\mu=0$, 


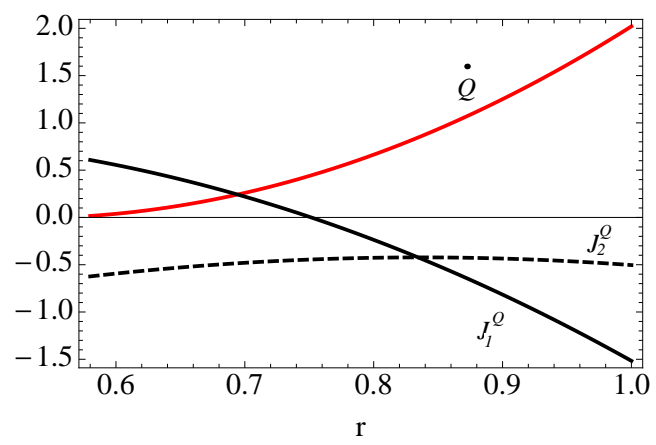

Figure 5: The flow $\dot{Q}>0$ and the currents $J_{1}^{Q}$ and $J_{2}^{Q}$ for $\lambda_{1}=-1.5$ and $\lambda_{2}=-3$.

this regime of energy transmutation in the junction can not be reached by linear response theory.

We have seen that for $\dot{Q}<0$ the junction can be compared to a heat engine. In analogy, one can attempt to interpret the system for $\dot{Q}>0$ as a refrigerator or a heat pump. A careful analysis shows that this possible only partially because there are subsets in $\mathcal{D}_{+}$where both heat currents have the same sign, i.e. they are both leaving or entering the heat reservoirs. A typical situation is shown in Fig. 5, where $J_{1}^{Q}$ and $J_{2}^{Q}$ become both negative (i.e. entering the heat reservoirs) for $r>0.75$, which is not the case of conventional refrigerators and heat pumps. For this reason the standard coefficients of performance for a refrigerator and a heat pump can not be applied in the whole domain $\mathcal{D}_{+}$. As already mentioned, this is our main motivation to introduce the efficiency $\widetilde{\eta}$ by (4.32), which has the advantage of working everywhere in $\mathcal{D}_{+}$.

Let us consider finally the entropy production. Substituting (5.42/5.43) in (4.33) one obtains

$$
\begin{aligned}
& \dot{S}=\frac{\left|\mathbb{S}_{12}\right|^{2}}{2 \pi r \beta_{1}}\left\{(1-r)\left[r^{2} \mathrm{Li}_{2}\left(-\mathrm{e}^{-\lambda_{2}}\right)-\mathrm{Li}_{2}\left(-\mathrm{e}^{-\lambda_{1}}\right)\right]\right. \\
& \left.+r\left(\lambda_{1}-\lambda_{2}\right)\left[r \ln \left(1+\mathrm{e}^{-\lambda_{2}}\right)-\ln \left(1+\mathrm{e}^{-\lambda_{1}}\right)\right]\right\}>0,
\end{aligned}
$$

confirming the general statement 24] about the entropy production in the LB state.

\subsection{Transport in the orbit $O_{\beta, \mu}$}

We consider here the quantum transport in the states $\left\{\Omega_{\beta, \mu}^{X}: X=P, T, P T\right\} \subset \mathcal{H}_{\mathrm{LB}}$. Using (3.16), the current expectation values in these states are simply expressed in terms of (4.26,4.27) as follows:

$$
\begin{aligned}
& P \quad: \quad J_{i}^{N} \longmapsto-J_{i}^{N} \quad J_{i}^{E} \longmapsto-J_{i}^{E}, \\
& T: J_{i}^{N} \longmapsto J_{i}^{N} \quad J_{i}^{E} \longmapsto-J_{i}^{E}, \\
& P T \quad: \quad J_{i}^{N} \longmapsto-J_{i}^{N} \quad J_{i}^{E} \longmapsto J_{i}^{E} .
\end{aligned}
$$


Let us observe in passing that the action of the charge conjugation

$$
C: J_{i}^{N} \longmapsto-J_{i}^{N} \quad J_{i}^{E} \longmapsto J_{i}^{E},
$$

coincides with the $P T$ operation (5.61). In fact, $C P T$ on the above currents is the identity transformation.

The minus sign appearing in some currents affects the quantum transport. Moreover, the entropy production $\dot{S}^{X}$ in the state $\Omega_{\beta, \mu}^{X}$ differs from that (5.58) in the LB state. In fact, combining (4.33) and (5.59) we conclude that $\dot{S}^{P} \leq 0$ for all values of the heat reservoir parameters $\left(\beta_{i}, \mu_{i}\right)$. We see that the state $\Omega_{\beta, \mu}^{P}$, which from the microscopic point of view is a well defined state of the system, violates the second law of thermodynamics and therefore has no admissible macroscopic behavior. The situation with the states $\Omega_{\beta, \mu}^{T}$ and $\Omega_{\beta, \mu}^{P T}$ is more subtle. The analysis shows that for these two states there exist domains in the whole parameter space $\left(\beta_{i}, \mu_{i}\right)$, where $\dot{S}^{T}>0$ and $\dot{S}^{P T}>0$. In these domains the quantum transport is consistent with the laws of thermodynamics. We consider for illustration the family of states $\left\{\Omega_{\beta, \mu}^{P T}: \lambda_{1}=\lambda_{2} \equiv \lambda\right\}$. The entropy production there is

$$
\dot{S}^{P T}=-\frac{\left|\mathbb{S}_{12}\right|^{2}}{2 \pi r \beta_{1}}(1-r)^{2}(1+r) \operatorname{Li}_{2}\left(-\mathrm{e}^{-\lambda}\right)>0
$$

Moreover

$$
\dot{Q}^{P T}(\lambda, \lambda, r)=\frac{\left|\mathbb{S}_{12}\right|^{2}}{2 \pi \beta_{1}^{2}}(1-r)^{2} \lambda \ln \left(1+\mathrm{e}^{-\lambda}\right)<0 \quad \text { for } \quad \lambda<0,
$$

and the efficiency of transforming heat into chemical potential energy is given by

$$
\eta^{P T}(\lambda, r)=\frac{(1-r) \lambda \ln \left(1+\mathrm{e}^{-\lambda}\right)}{\lambda \ln \left(1+\mathrm{e}^{-\lambda}\right)+(1+r) \mathrm{Li}_{2}\left(-\mathrm{e}^{-\lambda}\right)}, \quad \lambda<0 .
$$

This result resembles (5.54), but for a sign in the denumerator. The relative maximum is

$$
\eta_{\max }^{P T}(r)=\lim _{\lambda \rightarrow-\infty} \eta^{P T}(\lambda, r)=\frac{2(1-r)}{r+3} \neq \eta_{C},
$$

which coincides also with the efficiency at maximal chemical energy production

$$
\eta^{* P T}(r)=\frac{2(1-r)}{r+3}<\eta_{C A} .
$$

These results show that the efficiency in the state $\Omega_{\beta, \mu}^{P T}$ differs from that in $\Omega_{\beta, \mu}$. We will elaborate more on the value of $\eta^{* P T}$ few lines below.

Concerning the regime $\dot{Q}>0$, it is easy to deduce from (5.59) 5.61) that all four states in $O_{\beta, \mu}$ have the same efficiency $\widetilde{\eta}$ given by (5.57).

Summarizing, parity and time reversal have an important impact on the quantum transport and efficiency. Indeed, we have shown that there are regions in the parameter space of the states $\Omega_{\beta, \mu}^{T}$ and $\Omega_{\beta, \mu}^{P T}$, where the second law of thermodynamics is satisfied and the efficiency $\eta$ has a physically acceptable value, which differs from that in the LB state $\Omega_{\beta, \mu}$. 


\subsection{Comments about $\eta^{*}$}

It is instructive to compare now (5.54,5.67) with some exact results about the efficiency at maximal power obtained for other systems. Applying stochastic thermodynamics to a simple model of classical particle transport, the following explicit expression

$$
\eta_{c p}^{*}=\frac{\eta_{C}^{2}}{\eta_{C}-\left(1-\eta_{C}\right) \ln \left(1-\eta_{C}\right)}
$$

has been derived in [29]. The same expression has been obtained [28] for the Feynman's ratchet model as a heat engine. For a Brownian particle undergoing a Carnot cycle it has been found 31 that

$$
\eta_{B p}^{*}=\frac{2 \eta_{C}}{4-\eta_{C}}
$$

which, remarkably enough, coincides with the Schrödinger junction efficiency $\eta^{* P T}$ given by (5.67). The case of electron transport through a quantum dot has been treated in [32, 33].

It has been observed in [28, 29, 33] that away $(r<0.5)$ from the linear response regime, the efficiency (5.68) exceeds the Curzon-Ahlborn bound. One can wonder if a mechanism exists to enhance the efficiency (5.54) in the LB state $\Omega_{\beta, \mu}$ above $\eta_{C A}$ as well. This question attracted recently some attention [2], the proposal being to couple the system with an appropriate external potential. In [2] the effect of a classical magnetic field has been explored in the linear response approximation. We describe here an alternative, which simplifies our previous construction in [34] both from the technical and physical points of view. The main idea is based on the fact that in our general setting the heat reservoir dispersion relations $\omega_{i}$ need not to be equal and/or to coincide with the bulk dispersion relation $\omega$. So, let us perform the shift $\omega \longmapsto \omega-v$, which is equivalent to the introduction of a constant potential $V=-v$ in the bulk equation of motion (3.12). If one performs the same shift in $\omega_{i}$ the efficiency will not change. One can imagine however to screen the reservoirs $R_{i}$ from the potential $V$, thus keeping $\omega_{i}=k^{2} / 2 m$ invariant. This operation does not affect the particle currents $J_{i}^{N}$ which, according to (4.26), depend only on $\omega_{i}$. From (4.27) one infers however that the shift in $\omega$ modifies the energy transport in the following simple way

$$
J_{i}^{E} \longmapsto J_{i}^{E}-v J_{i}^{N}
$$

In the domain $\mathcal{D}_{-}$, which is still given by (5.46), one finds the efficiency

$$
\eta\left(\lambda_{1}, \lambda_{2} ; r, a\right)=\frac{\left(\lambda_{1}-r \lambda_{2}\right)\left[\ln \left(1+\mathrm{e}^{-\lambda_{1}}\right)-r \ln \left(1+\mathrm{e}^{-\lambda_{2}}\right)\right]}{\left(\lambda_{1}-a\right)\left[\ln \left(1+\mathrm{e}^{-\lambda_{1}}\right)-r \ln \left(1+\mathrm{e}^{-\lambda_{2}}\right)\right]-\left[\operatorname{Li}_{2}\left(-\mathrm{e}^{-\lambda_{1}}\right)-r^{2} \operatorname{Li}_{2}\left(-\mathrm{e}^{-\lambda_{2}}\right)\right]},
$$

with $a \equiv \beta_{1} v$ being dimensionless. The maximal efficiency is attained at $\lambda_{1}=\lambda_{2} \rightarrow+\infty$ and equals $\eta_{C}$ as before. For $\eta^{*}$ one finds instead

$$
\eta^{*}(r, a) \equiv \eta\left(\lambda^{*}, \lambda^{*} ; r, a\right)=\frac{(1-r) \lambda^{*} \ln \left(1+\mathrm{e}^{-\lambda^{*}}\right)}{\left(\lambda^{*}-a\right) \ln \left(1+\mathrm{e}^{-\lambda^{*}}\right)-(1+r) \operatorname{Li}_{2}\left(-\mathrm{e}^{-\lambda^{*}}\right)},
$$


which reproduces (5.54) for $a=0$. For the moment $a$ is a free real parameter, but the condition (4.36) is violated and the sign of $\dot{S}$ needs to be investigated. Imposing $\dot{S}>0$ for all $r \in[0,1]$, one obtains the constraint

$$
a<\frac{-\mathrm{Li}_{2}\left(-\mathrm{e}^{-\lambda^{*}}\right)}{\ln \left(1+\mathrm{e}^{-\lambda^{*}}\right)}=1,07122 \ldots \equiv a^{*} .
$$

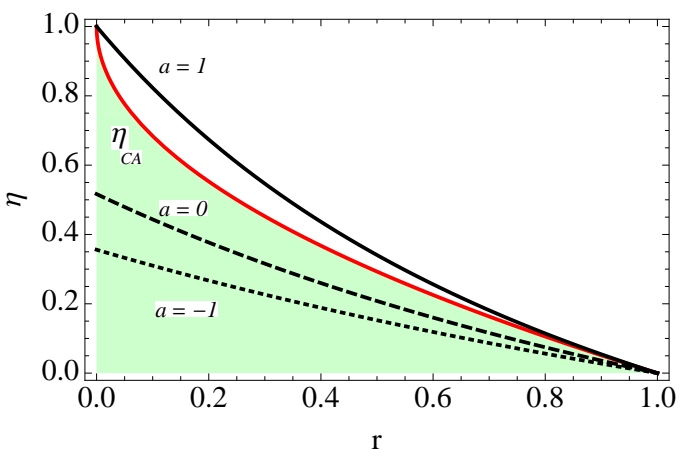

Figure 6: The efficiency $\eta^{*}(r, a)$ for different values of $a$ compared to $\eta_{C A}$.

It is easy to deduce from (5.72) that for $a \in\left(0, a^{*}\right)$ the efficiency $\eta^{*}(r, a)$ is enhanced above $\eta^{*}(r, 0)=\eta^{*}(r)$ given by (5.54). The opposite effect is observed for $a \in(-\infty, 0)$. This behavior of the efficiency is explained by the following intuitive physical argument. Since $\omega_{i}-\omega=a / \beta_{1}$, positive values of $a$ favor the particle emission from the heat reservoirs $R_{i}$ to the leads $L_{i}$, thus improving the efficiency. Negative values of $a$ are instead damping this emission, causing the opposite effect.

Fig. 6 illustrates the basic properties of $\eta^{*}(r, a)$. In particular, we see that for $a \sim 1$ the Curzon-Ahlborn bound is exceeded.

Finally, concerning the experimental realization of the above theoretical setup, one possibility could be to use the electric field produced by a wire with constant linear static charge distribution, which is located parallel at a finite distance from the two-terminal devise in Fig. 2. This charged wire produces a constant electric field along the devise. When the heat baths are screened by metallic boxes, the electric field provides the constant shift needed in the bulk dispersion relation.

\section{Junctions with three leads}

The new element in the treatment of the case with $n>2$ leads is that the transmission amplitudes $\left|\mathbb{S}_{i j}\right|^{2}$ do no longer factorize in front of the currents. For $n=3$ the transport properties depend on 9 parameters. The linear response approximation has been studied recently in [23, 35]. Here we pursue further the exact analysis of the scale invariant case for

$$
\beta_{1} \mu_{1}=\beta_{2} \mu_{2}=\beta_{3} \mu_{3} \equiv-\lambda, \quad r=\frac{\beta_{1}}{\beta_{2}}, \quad s=\frac{\beta_{1}}{\beta_{3}} .
$$


Using (4.39), one obtains from (4.40]4.41) the heat currents

$$
\begin{array}{r}
J_{1}^{Q}=\frac{1}{2 \pi \beta_{1}^{2}}\left\{\lambda \ln \left(1+\mathrm{e}^{-\lambda}\right)\left[\left|\mathbb{S}_{12}\right|^{2}(1-r)+\left|\mathbb{S}_{13}\right|^{2}(1-s)\right]\right. \\
\left.-\mathrm{Li}_{2}\left(-\mathrm{e}^{-\lambda}\right)\left[\left|\mathbb{S}_{12}\right|^{2}\left(1-r^{2}\right)+\left|\mathbb{S}_{13}\right|^{2}\left(1-s^{2}\right)\right]\right\}, \\
J_{2}^{Q}=\frac{1}{2 \pi \beta_{1}^{2}}\left\{r \lambda \ln \left(1+\mathrm{e}^{-\lambda}\right)\left[\left|\mathbb{S}_{23}\right|^{2}(r-s)+\left|\mathbb{S}_{12}\right|^{2}(r-1)\right]\right. \\
\left.-\operatorname{Li}_{2}\left(-\mathrm{e}^{-\lambda}\right)\left[\left|\mathbb{S}_{23}\right|^{2}\left(r^{2}-s^{2}\right)+\left|\mathbb{S}_{12}\right|^{2}\left(r^{2}-1\right)\right]\right\}, \\
J_{3}^{Q}=\frac{1}{2 \pi \beta_{1}^{2}}\left\{s \lambda \ln \left(1+\mathrm{e}^{-\lambda}\right)\left[\left|\mathbb{S}_{23}\right|^{2}(s-r)+\left|\mathbb{S}_{13}\right|^{2}(s-1)\right]\right. \\
\left.-\operatorname{Li}_{2}\left(-\mathrm{e}^{-\lambda}\right)\left[\left|\mathbb{S}_{23}\right|^{2}\left(s^{2}-r^{2}\right)+\left|\mathbb{S}_{13}\right|^{2}\left(s^{2}-1\right)\right]\right\} .
\end{array}
$$

For the entropy production and heat flow on gets

$$
\begin{gathered}
\dot{S}=-\frac{\mathrm{Li}_{2}\left(-\mathrm{e}^{-\lambda}\right)}{2 \pi s r \beta_{1}}\left[\left|\mathbb{S}_{12}\right|^{2} s(1+r)(1-r)^{2}+\left|\mathbb{S}_{13}\right|^{2} r(1+s)(1-s)^{2}+\left|\mathbb{S}_{23}\right|^{2}(r+s)(r-s)^{2}\right]>0 \\
\dot{Q}=-\frac{1}{2 \pi \beta_{1}^{2}} \lambda \ln \left(1+\mathrm{e}^{-\lambda}\right)\left[\left|\mathbb{S}_{12}\right|^{2}(1-r)^{2}+\left|\mathbb{S}_{13}\right|^{2}(1-s)^{2}+\left|\mathbb{S}_{23}\right|^{2}(r-s)^{2}\right]
\end{gathered}
$$

As expected, the entropy production in the LB state is always positive. For $\lambda>0$ one has $\dot{Q}<0$ and one can study the efficiency (4.31) of transforming heat to chemical potential energy. In order to do that one has to determine first the sign of the currents (6.75)6.77). Without loss of generality one can assume that

$$
r<1, \quad s<1, \quad r<s,
$$

which implies the following ordering $T_{2}<T_{3}<T_{1}$ among the temperatures of the heat reservoirs. For simplifying the analysis we assume to end of this section that $\left|\mathbb{S}_{13}\right|^{2}=0$, devoting the appendix B to the case of generic transmission amplitudes. Then (6.75, 6.77) imply $J_{1}^{Q}>0, J_{2}^{Q}<0, J_{3}^{Q}>0$ for $\lambda>0$. With this information one obtains from (4.31)

$$
\begin{gathered}
\eta(\lambda ; r, s)= \\
\frac{\lambda \ln \left(1+\mathrm{e}^{-\lambda}\right)\left[\left|\mathbb{S}_{12}\right|^{2}(1-r)^{2}+\left|\mathbb{S}_{23}\right|^{2}(s-r)^{2}\right]}{\lambda \ln \left(1+\mathrm{e}^{-\lambda}\right)\left[\left|\mathbb{S}_{12}\right|^{2}(1-r)+\left|\mathbb{S}_{23}\right|^{2} s(s-r)\right]-\mathrm{Li}_{2}\left(-\mathrm{e}^{-\lambda}\right)\left[\left|\mathbb{S}_{12}\right|^{2}\left(1-r^{2}\right)+\left|\mathbb{S}_{23}\right|^{2}\left(s^{2}-r^{2}\right)\right]} .
\end{gathered}
$$

The relative maximum is

$$
\eta_{\max }(r, s)=\lim _{\lambda \rightarrow+\infty} \eta(\lambda ; r, s)=1-r \frac{\left|\mathbb{S}_{12}\right|^{2}(1-r)+\left|\mathbb{S}_{23}\right|^{2}(s-r)}{\left|\mathbb{S}_{12}\right|^{2}(1-r)+\left|\mathbb{S}_{23}\right|^{2} s(s-r)},
$$


which suggests to introduce an effective $r$-parameter

$$
r_{\text {eff }}(s)=r \frac{\left|\mathbb{S}_{12}\right|^{2}(1-r)+\left|\mathbb{S}_{23}\right|^{2}(s-r)}{\left|\mathbb{S}_{12}\right|^{2}(1-r)+\left|\mathbb{S}_{23}\right|^{2} s(s-r)} \leq 1 \quad \text { for } \quad r<1, r<s
$$

with the following physical meaning. The hotter reservoirs of our system are $R_{1}$ and $R_{3}$ because $T_{1}>T_{2}$ and $T_{3}>T_{2}$. Both $R_{1}$ and $R_{3}$ communicate with the cold reservoir $R_{2}$ via $\mathbb{S}_{12}$ and $\mathbb{S}_{23}$. Suppose now we replace $R_{1}$ and $R_{3}$ with one heat bath $R^{\prime}$ and ask about its temperature $T^{\prime}$, which gives the same efficiency. It turns out that the answer is $T^{\prime}=T_{2} / r_{\text {eff }}$. In fact, one can express the 3-lead efficiency (6.81) in terms of the 2-lead formula (5.51) simply as

$$
\eta(\lambda ; r, s)=\eta\left(\lambda, \lambda ; r_{\mathrm{eff}}(s)\right) .
$$

We turn now to the regime of heat production $\dot{Q}>0$ which, according to (6.79), takes place for $\lambda<0$. The corresponding efficiency is defined by (4.32). Let us introduce the parameters

$$
u=\frac{\mu_{2}}{\mu_{1}}, \quad v=\frac{\mu_{3}}{\mu_{1}} .
$$

Because of (6.74), the conditions (6.80) can be rewritten in the form

$$
u<1, \quad v<1, \quad u<v .
$$

One can show now that $\mu_{1} J_{1}^{N}>0, \mu_{2} J_{2}^{N}<0, \mu_{3} J_{3}^{N}>0$. Therefore, using (4.32) one obtains

$$
\widetilde{\eta}(u, v)=1-u \frac{\left|\mathbb{S}_{12}\right|^{2}(1-u)+\left|\mathbb{S}_{23}\right|^{2}(v-u)}{\left|\mathbb{S}_{12}\right|^{2}(1-v)+\left|\mathbb{S}_{23}\right|^{2} v(v-u)} .
$$

The effective $u$-parameter now reads

$$
u_{\mathrm{eff}}(v)=u \frac{\left|\mathbb{S}_{12}\right|^{2}(1-u)+\left|\mathbb{S}_{23}\right|^{2}(v-u)}{\left|\mathbb{S}_{12}\right|^{2}(1-v)+\left|\mathbb{S}_{23}\right|^{2} v(v-u)} \leq 1 \quad \text { for } \quad u<1, u<v
$$

and

$$
\widetilde{\eta}(u, v)=\widetilde{\eta}\left(u_{\mathrm{eff}}(v)\right),
$$

whose right hand side is given by the first line (since $u<1$ ) of the two-lead expression (5.57).

We refer to appendix $\mathrm{B}$ for the analysis of the general case, in which all three transmission amplitudes are nontrivial.

\section{Bosonic junctions}

We illustrate in this section the influence of the statistics on the transport and efficiency of the Schrödinger junction. Substituting in (4.26 4.27) the Fermi distribution $d_{j}(k)$ with the Bose one $b_{j}(k)$, the corresponding integrands develop a singularity at $k^{2}=2 m \mu_{i}$. This singularity signals condensation like phenomena, whose consideration is beyond the scope of 
the present paper. For this reason we assume in this section $\mu_{i}<0$. Focussing on the case $n=2$, the bosonic counterparts of (5.42,5.43,5.45,5.58) are

$$
\begin{gathered}
J_{1}^{N}=-J_{2}^{N}=\frac{\left|\mathbb{S}_{12}\right|^{2}}{2 \pi \beta_{1}}\left[-\ln \left(1-\mathrm{e}^{-\lambda_{1}}\right)+r \ln \left(1-\mathrm{e}^{-\lambda_{2}}\right)\right] \\
J_{1}^{E}=-J_{2}^{E}=\frac{\left|\mathbb{S}_{12}\right|^{2}}{2 \pi \beta_{1}^{2}}\left[\operatorname{Li}_{2}\left(\mathrm{e}^{-\lambda_{1}}\right)-r^{2} \operatorname{Li}_{2}\left(\mathrm{e}^{-\lambda_{2}}\right)\right] \\
\dot{Q}^{(b)}\left(\lambda_{1}, \lambda_{2}, r\right)=\frac{\left|\mathbb{S}_{12}\right|^{2}}{2 \pi \beta_{1}^{2}}\left(\lambda_{1}-r \lambda_{2}\right)\left[\ln \left(1-\mathrm{e}^{-\lambda_{1}}\right)-r \ln \left(1-\mathrm{e}^{-\lambda_{2}}\right)\right] \\
\dot{S}^{(b)}=\frac{\left|\mathbb{S}_{12}\right|^{2}}{2 \pi r \beta_{1}}\left\{(1-r)\left[\operatorname{Li}_{2}\left(\mathrm{e}^{-\lambda_{1}}\right)-r^{2} \operatorname{Li}_{2}\left(\mathrm{e}^{-\lambda_{2}}\right)\right]+r\left(\lambda_{1}-\lambda_{2}\right)\left[\ln \left(1-\mathrm{e}^{-\lambda_{1}}\right)-r \ln \left(1-\mathrm{e}^{-\lambda_{2}}\right)\right]\right\}
\end{gathered}
$$

where one should keep in mind that $\lambda_{i} \equiv-\beta_{i} \mu_{i}>0$. The apex $(b)$ in (7.92,7.93) and below is added in order to distinct the bosonic from the fermionic expressions. The domains $\mathcal{D}_{\mp}^{(b)}$, where $\dot{Q}^{(b)} \lessgtr 0$, can be determined like in fermion case. One has

$$
\mathcal{D}_{-}^{(b)}=D_{1}^{(b)} \cup D_{2}^{(b)}
$$

with

$$
\begin{gathered}
D_{1}^{(b)}=\left\{0<\lambda_{1} \leq \lambda_{2}, 0 \leq r<r_{1}\right\}, \\
D_{2}^{(b)}=\left\{0<\lambda_{2}<\lambda_{1}, 0 \leq r<r_{2}\right\}, \\
r_{1}=\frac{\lambda_{1}}{\lambda_{2}}, \quad r_{2}=\frac{\ln \left(1-\mathrm{e}^{-\lambda_{1}}\right)}{\ln \left(1-\mathrm{e}^{-\lambda_{2}}\right)} .
\end{gathered}
$$

In $\mathcal{D}_{-}^{(b)}$ one finds the bosonic efficiency

$$
\eta^{(b)}\left(\lambda_{1}, \lambda_{2}, r\right)=\frac{\left(\lambda_{1}-r \lambda_{2}\right)\left[r \ln \left(1-\mathrm{e}^{-\lambda_{2}}\right)-\ln \left(1-\mathrm{e}^{-\lambda_{1}}\right)\right]}{\lambda_{1}\left[r \ln \left(1-\mathrm{e}^{-\lambda_{2}}\right)-\ln \left(1-\mathrm{e}^{-\lambda_{1}}\right)\right]+\left[\mathrm{Li}_{2}\left(\mathrm{e}^{-\lambda_{1}}\right)-r^{2} \operatorname{Li}_{2}\left(\mathrm{e}^{-\lambda_{2}}\right)\right]} .
$$

In the overlap of $\mathcal{D}_{-}$and $\mathcal{D}_{-}^{(b)}$ one can compare (7.98) to the fermion efficiency (5.51). For the same values of $\lambda_{i}$ in $D_{1}^{(b)}=D_{1}$ one finds that the bosonic efficiency exceeds the fermionic one. The same holds for the amount of heat transformed in chemical energy. This feature persists in the overlap of $D_{2}^{(b)}$ with $D_{2}$, except in the neighborhood of $r_{2}$, where one has the opposite behavior. The maximal bosonic efficiency is obtained for $\lambda_{1}=\lambda_{2} \equiv \lambda \rightarrow+\infty$ and coincides with the Carnot efficiency,

$$
\eta_{\max }^{(b)}(r)=\lim _{\lambda \rightarrow+\infty} \eta^{(b)}(\lambda, \lambda, r)=1-r \equiv \eta_{C} .
$$

Let us derive now the bosonic efficiency at maximal chemical energy production. The maximum of the function $-\dot{Q}^{(b)}\left(\lambda_{1}, \lambda_{2}, r\right)$ is reached at $\lambda_{1}=\lambda_{2} \equiv \lambda_{b}^{*}$, where

$$
\lambda_{b}^{*}-\left(1-\mathrm{e}^{\lambda_{b}^{*}}\right) \ln \left(1-\mathrm{e}^{-\lambda_{b}^{*}}\right)=0 .
$$




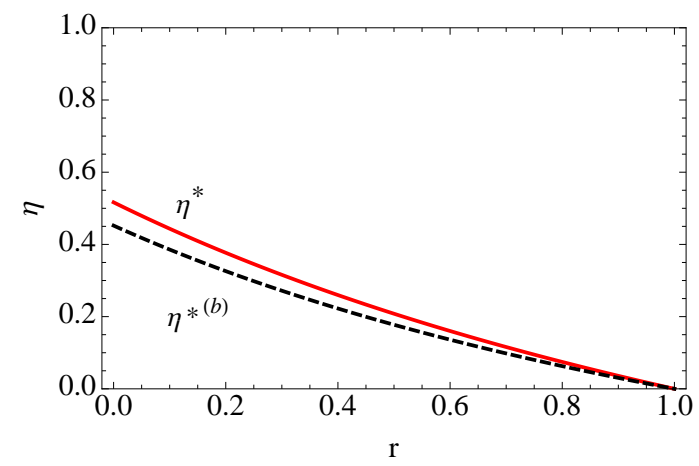

Figure 7: Comparing the bosonic and fermionic efficiencies $\eta^{*(b)}$ and $\eta^{*}$.

The solution of (7.100) is $\lambda_{b}^{*}=0.69314 \ldots$ and the efficiency reads

$$
\eta^{*(b)}(r) \equiv \eta^{(b)}\left(\lambda_{b}^{*}, \lambda_{b}^{*}, r\right)=\frac{(1-r) \lambda_{b}^{*} \ln \left(1-\mathrm{e}^{-\lambda_{b}^{*}}\right)}{\lambda_{b}^{*} \ln \left(1-\mathrm{e}^{-\lambda_{b}^{*}}\right)-(1+r) \mathrm{Li}_{2}\left(\mathrm{e}^{-\lambda_{b}^{*}}\right)} .
$$

The study of (7.101) reveals that at maximal chemical energy production the bosonic junction is slightly less efficient then the fermion one, as shown in Fig. 7 . The enhancement mechanism for fermions, described in section 5.3, applies to bosonic junctions as well.

We conclude by observing that in the regime $\dot{Q}^{(b)}>0$ the bosonic efficiency $\widetilde{\eta}^{(b)}$ of transforming chemical energy in heat precisely coincides with the fermionic one (5.57).

\section{Conclusions}

We described in this paper a basic process of transformation of heat in chemical potential energy and vice versa, which takes place in systems away from equilibrium. The phenomenon is universal and stems from the fact that even if the total energy of the system is conserved, the heat and chemical potential energies are in general not separately conserved. Both directions of the process of energy transmutation are characterized by their own efficiency coefficient. The specific features of the phenomenon depend on the particle statistics and on the choice of nonequilibrium state. We illustrated this fact by studying the fermionic and bosonic Schrödinger junctions in the LB state and its orbit under parity and time reversal. The relative quantum transport depends in a complicated nonlinear way on the temperatures and chemical potentials, which parametrize the nonequilibrium states. In order to control these characteristics of the system, we avoided the use of any approximation and in particular, of the linear response theory. Assuming that the interaction which drives the system away from equilibrium is scale invariant, we described the quantum transport exactly and derived the explicit expressions of the efficiency coefficients.

Exploring the orbit of the LB state under parity and time reversal transformations, we have shown that space-time symmetries have an essential impact on the quantum transport and efficiencies. Concerning the internal symmetries, our model has a global $U(1)$-symmetry associated with the particle number. For systems with a larger internal symmetry group, 
the process of energy transmutation becomes even more involved, due to the presence of several types of chemical potentials. In this case the Landauer-Büttiker state is induced not by a Gibbs state, but by a generalized Gibbs ensemble [36] generated by a complete set of commuting charges of the extended symmetry group. The study of such nonequilibrium states may provide an important insight in the role of internal symmetries in quantum transport.

\section{Acknowledgments:}

M.M. would like to thank the Laboratoire de Physique Théorique d'Annecy-le-Vieux for the kind hospitality during the preparation of the manuscript.

\section{A Contact with linear response theory}

We show here that the exact efficiency (5.51) reproduces in the linear response regime the result of [2]. The meeting point of the two frameworks is the Onsager matrix $X$ [21]. Setting

$$
\beta_{1}=\beta, \quad \beta_{2}=\beta+\delta \beta, \quad \mu_{1}=\mu, \quad \mu_{2}=\mu+\delta \mu, \quad \lambda=-\beta \mu,
$$

the entries $X_{i j}$ of $X$ are defined by [21]

$$
\begin{aligned}
-J_{1}^{N} & =X_{11} \beta \delta \mu+X_{12} \delta \beta+\cdots, \\
J_{1}^{Q}=J_{1}^{E}-\mu J_{1}^{N} & =X_{21} \beta \delta \mu+X_{22} \delta \beta+\cdots,
\end{aligned}
$$

where the dots stand for higher orders of the expansion in $\delta \mu$ and $\delta \beta$. For the Onsager matrix of our system with scale invariant interaction in the junction one gets from [2]

$$
\begin{aligned}
& X_{11}=\frac{\left|\mathbb{S}_{12}\right|^{2}}{2 \pi \beta} \frac{1}{1+\mathrm{e}^{\lambda}}, \\
& X_{12}=X_{21}=-\frac{\left|\mathbb{S}_{12}\right|^{2}}{2 \pi \beta^{2}}\left[\frac{\lambda}{1+\mathrm{e}^{\lambda}}+\ln \left(1+\mathrm{e}^{-\lambda}\right)\right], \\
& X_{22}=\frac{\left|\mathbb{S}_{12}\right|^{2}}{2 \pi \beta^{2}}\left[\frac{\lambda^{2}}{1+\mathrm{e}^{\lambda}}+2 \lambda \ln \left(1+\mathrm{e}^{-\lambda}\right)-\mathrm{Li}_{2}\left(-\mathrm{e}^{-\lambda}\right)\right] .
\end{aligned}
$$

Moreover, according to [2] the linear response efficiency $\eta_{\mathrm{LR}}$ is given by

$$
\eta_{\mathrm{LR}}=\frac{-\left(X_{11} \beta \delta \mu+X_{12} \delta \beta\right) \delta \mu}{X_{21} \beta \delta \mu+X_{22} \delta \beta} .
$$

Inserting (A.104) in (A.105) and expanding in $\delta \mu$ and $\delta \beta$ one obtains to the first order

$$
\begin{aligned}
\eta_{\mathrm{LR}}= & \frac{\mathrm{e}^{-\lambda}}{\left(1+\mathrm{e}^{-\lambda}\right) \ln \left(1+\mathrm{e}^{-\lambda}\right)+\lambda \mathrm{e}^{-\lambda}} \beta \delta \mu- \\
& \frac{\left(1+\mathrm{e}^{-\lambda}\right) \ln ^{2}\left(1+\mathrm{e}^{-\lambda}\right)+2 \mathrm{e}^{-\lambda} \mathrm{Li}_{2}\left(-\mathrm{e}^{-\lambda}\right)}{\beta\left[\left(1+\mathrm{e}^{-\lambda}\right) \ln \left(1+\mathrm{e}^{-\lambda}\right)+\lambda \mathrm{e}^{-\lambda}\right]^{2}} \delta \beta+\cdots .
\end{aligned}
$$


This result coincides precisely with the expansion of the exact efficiency (5.51) (expressed in terms of the variables (A.102) $)$ to the first order in $\delta \mu$ and $\delta \beta$, which concludes the proof. The above argument has a direct generalization to the case $n>2$.

\section{B The 3-lead junction with generic $\mathbb{S}$-matrix ampli- tudes}

Combining (6.75, 6.76) with (6.80), we conclude that $J_{1}^{Q}>0$ and $J_{2}^{Q}<0$. So, one is left with the study of the sign of $J_{3}^{Q}$ given by (6.77). The coefficients of the logarithm and the polylogarithm are both positive when

$$
s>\frac{r\left|\mathbb{S}_{23}\right|^{2}+\left|\mathbb{S}_{13}\right|^{2}}{\left|\mathbb{S}_{23}\right|^{2}+\left|\mathbb{S}_{13}\right|^{2}} \equiv s_{1}, \quad s^{2}>\frac{r^{2}\left|\mathbb{S}_{23}\right|^{2}+\left|\mathbb{S}_{13}\right|^{2}}{\left|\mathbb{S}_{23}\right|^{2}+\left|\mathbb{S}_{13}\right|^{2}} \equiv s_{2}^{2} .
$$

Using (6.80) it is not difficult to show that $s_{2}>s_{1}>r$. Therefore,

$$
\begin{array}{ll}
J_{3}^{Q}<0, & \text { for } \quad r<s<s_{1}, \\
J_{3}^{Q}>0, & \text { for } \quad s_{2}<s<1 .
\end{array}
$$

For $s \in\left(s_{1}, s_{2}\right)$ the sign of $J_{3}^{Q}$ depends on $\lambda$ as well. In this way one finds

$$
\eta(\lambda ; r, s)= \begin{cases}\frac{A}{B_{1}} & \text { for } \quad r<s<s_{1} \\ \frac{A}{B_{2}} & \text { for } \quad s_{2}<s<1\end{cases}
$$

where

$$
\begin{gathered}
A=\lambda \ln \left(1+\mathrm{e}^{-\lambda}\right)\left[\left|\mathbb{S}_{12}\right|^{2}(1-r)^{2}+\left|\mathbb{S}_{13}\right|^{2}(1-s)^{2}+\left|\mathbb{S}_{23}\right|^{2}(s-r)^{2}\right] \\
B_{1}=\lambda \ln \left(1+\mathrm{e}^{-\lambda}\right)\left[\left|\mathbb{S}_{12}\right|^{2}(1-r)+\left|\mathbb{S}_{13}\right|^{2}(1-s)\right]- \\
\operatorname{Li}_{2}\left(-\mathrm{e}^{-\lambda}\right)\left[\left|\mathbb{S}_{12}\right|^{2}\left(1-r^{2}\right)+\left|\mathbb{S}_{13}\right|^{2}\left(1-s^{2}\right)\right]
\end{gathered}
$$

and

$$
\begin{gathered}
B_{2}=\lambda \ln \left(1+\mathrm{e}^{-\lambda}\right)\left[\left|\mathbb{S}_{12}\right|^{2}(1-r)+\left|\mathbb{S}_{13}\right|^{2}(1-s)^{2}+\left|\mathbb{S}_{23}\right|^{2} s(s-r)\right]- \\
\operatorname{Li}_{2}\left(-\mathrm{e}^{-\lambda}\right)\left[\left|\mathbb{S}_{12}\right|^{2}\left(1-r^{2}\right)+\left|\mathbb{S}_{23}\right|^{2}\left(s^{2}-r^{2}\right)\right] .
\end{gathered}
$$

\section{References}

[1] A. Polkovnikov, K. Sengupta, A. Silva and M. Vengalatorre, Rev. Mod. Phys. 83, 863 (2012). 
[2] C. Benenti, G. Casati, T. Prosen and K. Saito, Fundamental aspects of steady state heat to work conversion, arXiv: 1311.4430.

[3] R. Landauer, IBM J. Res. Dev. 1, 233 (1957); Philos. Mag. 21, 863 (1970).

[4] M. Büttiker, Phys. Rev. Lett. 57,1761 (1986); IBM J. Res. Dev. 32, 317 (1988).

[5] M. Mintchev, J. Phys. A 44, 415201 (2011).

[6] G. L. Eyink, J. L. Lebowitz and H. Spohn, J. Stat. Phys. 83, 385 (1996).

[7] D. Ruelle, J. Stat. Phys. 98, 57 (2000).

[8] L. Bertini, A. D. Sole, D. Gabrielli, G. Jona-Lasinio and C. Landim, Phys. Rev. Lett. 87, 040601 (2001).

[9] V. Jaksic and C. A. Pillet, J. Stat. Phys. 108, 787 (2002).

[10] S. Sasa and H. Tasaki, J. Stat. Phys. 125, 125 (2006).

[11] O. Bratteli and D. W. Robinson, Operator Algebras and Quantum Statistical Mechanics 2, (Springer, 1996).

[12] A. Liguori, M. Mintchev and L. Zhao, Commun. Math. Phys. 194, 569 (1998).

[13] M. Mintchev, E. Ragoucy and P. Sorba, J. Phys. A 36, 10407 (2003).

[14] M. Mintchev and P. Sorba, J. Stat. Mech. 0407, P001 (2004).

[15] M. Mintchev and P. Sorba, J. Phys. A 46, 095006 (2013).

[16] V. Kostrykin and R. Schrader, Fortschr. Phys. 48, 703 (2000).

[17] M. Harmer, J. Phys. A 33, 9015 (2000).

[18] P. Kuchment, arXiv:0802.3442 [math-ph].

[19] B. Bellazzini, M. Mintchev and P. Sorba, Phys. Rev. B 82, 195113 (2010).

[20] B. Bellazzini, M. Burrello, M. Mintchev and P. Sorba, Proc. Symp. Pure Math. 77, 639 (2008), arXiv:0801.2852 [hep-th].

[21] H. B. Callen, Thermodynamics and an Introduction to Thermostatistics, (John Wiley and Sons, 1960).

[22] Sivan and Imry, Phys. Rev. B. 33, 551 (1986).

[23] F. Mazza, R. Bosisio, G. Benenti, V. Giovannetti, R, Fazio and F. Taddei, New J. Phys. 16, 085001 (2014). 
[24] G. Nenciu, J. Math. Phys. 48, 033302 (2007).

[25] P. Calabrese, M. Mintchev and E. Vicari, J. Phys. A 45, 105206 (2012).

[26] F. Curzon and B. Ahlborn, Am. J. Phys. 43, 22 (1975).

[27] C. Van den Broeck, Phys. Rev. Lett. 95, 190602 (2005).

[28] Z. C. Tu, J. Phys. A 41, 312003 (2008).

[29] C. Van den Broeck and K. Lindenberg, Phys. Rev. E 86, 041144 (2012).

[30] B. Sothmann and M. Büttiker, Europhys. Lett. 99, 27001 (2012).

[31] T. Schmiedl and U. Seifert, Europhys. Lett. 81, 20003 (2008).

[32] M. Esposito, K. Lindenberg and C. Van den Broeck, Europhys. Lett. 85, 60010 (2009).

[33] B. Sothmann, R. Sánchez and A. N. Jordan, Thermoelectric energy harvesting with quantum dots, arXiv: 1406.5329.

[34] M. Mintchev, L. Santoni and P. Sorba, Thermoelectric efficiency in critical quantum junctions, arXiv:1310.2392.

[35] O. Entin-Wohlman and A. Aharony, Phys. Rev. B 85, 085401 (2012).

[36] E. T. Jaynes, Phys. Rev. 106, 620 (1957). 